\title{
Conducta sexual de riesgo y comunicación familiar en adolescentes de 3ero, 4to y 5to año del nivel secundario de una I.E. estatal de la ciudad de lquitos
}

\section{Risky sexual behavior and family communication in adolescents of 3rd, 4th and 5th year of the secondary level of an I.E. state of the city of lquitos}

\author{
Keshia Gamboa Pumazoncco', Keila Ysuiza Rodriguez²
}

\begin{abstract}
RESUMEN
Objetivo: determinar la asociación entre la conducta sexual de riesgo y comunicación familiar en adolescentes de 3ro, 4to y 5to año del nivel secundario de una I. E. estatal de la ciudad de Iquitos. Metodología: diseño no experimental, de corte transversal y alcance correlacional. Se llevó a cabo en un grupo de 500 adolescentes entre 13 y 19 años. Los instrumentos usados fueron el cuestionario Conducta Sexual de Riesgo (CSR) por Apaza (2018) y la Escala de comunicación Padres-Adolescentes (PAC) de Barnes y Olson (1982) adaptado en el Perú por Bueno, Tomas y Araujo (1998). Resultados: los resultados obtenidos demuestran que no existe asociación significativa entre el nivel de comunicación con el padre y las conductas sexuales de riesgo. En cambio, se aprecia que el nivel de comunicación con la madre se asocia significativamente con la edad de inicio sexual $(X 2=2.303 ; p<.05)$, con la cantidad de parejas sexuales $(X 2=8.758 ; p<.01)$ y uso de preservativo $(X 2=3.803 ; p<.05)$ como medida de protección. Conclusiones: a mayor comunicación menor probabilidad en conducta sexual de riesgo.
\end{abstract}

Palabras clave: Conducta sexual de riesgo, comunicación familiar, adolescentes

\begin{abstract}
Objective: to determine the association between risky sexual behavior and family communication in adolescents of 3rd, 4th and 5th year of the secondary level of a state I.E. of the city of Iquitos. Methodology: non-experimental design, cross-sectional and correlational scope. It was carried out in a group of 500 adolescents between 13 and 19 years old. The instruments used were the questionnaire Sexual Risk Conduct (CSR) by Apaza (2018) and the Parent-Adolescent Communication Scale (PAC) by Barnes and Olson (1982) adapted in Peru by Bueno, Tomas and Araujo (1998). Results: the results obtained show that there is no significant association between the level of communication with the father and risky sexual behaviors. On the other hand, it can be seen that the level of communication with the mother is significantly associated with the age of sexual initiation $(X 2=2.303 ; p<.05)$, with the number of sexual partners $(X 2=8.758 ; p<.01)$ and use of condoms $(X 2=3.803 ; p<.05)$ as a protection measure. Conclusions: the greater the communication, the lower the probability of risky sexual behavior.
\end{abstract}

Keywords: Risky sexual behavior, Family communication, Adolescents.

${ }^{1}$ UGEL Contamana, Ucayali, Perú. Orcid ID: 0000-0001-8607-3670 ${ }^{2}$ UGEL Contamana, Ucayali, Perú. Orcid ID: 0000-0002-9887-0527 


\section{INTRODUCCIÓN}

En las últimas generaciones la maduración sexual en los adolescentes, es cada vez más temprana y sobre todo la participación es aún más activa en la esfera social, cultural y económica. Por ende, los problemas sexuales de riesgo se han convertido en un problema para la sociedad actual; tener sexo a temprana edad está asociado a un sexo no seguro, debido a que, existe falta de conocimiento e información, acceso a los métodos anticonceptivos, y casi en la mayoría de los adolescentes suelen iniciar también su relación sexual bajo los efectos del alcohol y drogas, esto imposibilitándolos a tomar decisiones estables y sobre todo a resistir la presión de la pareja. (Rosales e Irigoyen, 2013).

De la misma manera, la Organización Mundial de la Salud (2016) hace referencia que, cada día más de 1 millón de personas adquieren una infección de trasmisión sexual. A su vez, anualmente, unos 357 millones de personas contraen algunas de las cuatro ITS; clamidiasis (131 millones), gonorrea (78 millones), sífilis (5.6 millones) o tricomoniasis (143 millones). Asimismo, el porcentaje de personas con infección genital por el VHS (herpes) prevalece los 500 millones. Por lo tanto, en el mundo hay más de 290 millones de mujeres víctimas, infectadas con el virus de papiloma humano (VPH), una de las ITS más comunes.

Por otro lado, por Lavielle et al. (2014) indican la relación entre conducta de riesgo sexual y comunicación familiar, obteniendo como resultado que el $63.8 \%$ de los y las adolescentes presenta satisfacción y comunicación familiar, lo que se consideraba como familia nuclear, no obstante, un $21.7 \%$ provenía de un conjunto de familia monoparental. Sin embargo, en la forma o interacción de la comunicación y la estructura familiar de los adolescentes que han iniciado y no han iniciado su actividad sexual, un (23.9\%) se dio a conocer que los adolescentes presentaban una comunicación familiar disfuncional iniciando su vida sexual, asimismo el (27.4\%) de las y los adolescentes evidenciaban una comunicación funcional. También, el $33.8 \%$ de los adolescentes que habían tenido relaciones sexuales, provenía de una familia monoparental, $24.9 \%$ de una familia nuclear y $19.6 \%$, de una familia extensa. Es decir que, la iniciación temprana de la vida sexual fue más frecuente en los adolescentes con familias en la cual la expresión de afecto era escaza.
Fernándezetal. (2017)ejecuto un estudioen Costa Rica, sobre las actitudes hacia la comunicación sexual entre madres, padres y adolescentes. Con una muestra de 916 personas madres y padres como adolescentes de 13 a 17 años. Utilizaron para tal fin el Parent-Teen Sexual Communication Scalem, esta escala mide la comunicación entre padres, madres e hijos (as) sobre la actividad sexual, reproducción, contraceptivos, infecciones de transmisión sexual, protección de estas enfermedades, uso del condón, presión de grupo y presión sexual por parte de otros adolescentes. Se empleó un diseño experimental con seguimiento longitudinal. Cuyos resultados obtuvieron que el $15 \%$ de adolescentes se encontraban activos sexualmente, sin embargo, se encontró que los adolescentes tienen mejor disposición que los padres y madres para hablar sobre sexualidad, por otro lado, los padres, madres y adolescentes reportaron algún grado de dificultad e incomodidad al hablar sobre métodos específicos de prevención, esto llevándolos a destacar una necesidad de incorporar intervenciones con adolescentes y padres sobre tema de salud mental.

En Tacna, Gómez (2013) realizó un estudio en la Institución educativa Cesar Cohaila Tamayo con el objetivo de determinar la relación entre los conocimientos y las actitudes frente a las conductas sexuales de riesgo en adolescentes. El estudio fue de tipo cuantitativo, nivel aplicativo y método descriptivo de corte transversal. La muestra poblacional fue de 162 adolescentes del 3ro, 4to y 5to año de nivel secundario. Obteniendo como resultado que el $51,2 \%$ de los adolescentes expresaron una actitud de indiferencia hacia las conductas sexuales de riesgo, sin embargo, el (31,5\%) presentaron una conducta de rechazo y solo el $(17,3 \%)$ de adolescentes expresaron una actitud de aceptación frente a las conductas sexuales de riesgo. Llegando a la conclusión que la mayoría de los adolescentes tienen un nivel de conocimiento "medio" sobre las infecciones de transmisión sexual y la mayoría tienen actitudes de "indiferencia" frente a las conductas sexuales de riesgo.

\section{Aspectos teóricos de la conducta sexual de riesgo}

Definición: Valle et al. (2011) las conductas sexuales de riesgo es la exposición de un individuo a situaciones que puede producir daños 
irreparables en su salud, que en ocasiones involucra a otra persona. Las infecciones de trasmisión sexual y los embarazos no deseados constituyen los dos principales problemas asociados con la conducta sexual de riesgo.

Aspectos teóricos: De acuerdo a la propuesta por Jessor (1991 y 1993), reconoce que la conducta de riesgo del adolescente, bajo una nueva perspectiva. La teoría de la conducta de riesgo de los adolescentes se caracteriza por ser compleja y esto llega a explicar las conductas adictivas. Considerando factores de riesgo y protección, conductas de riesgo y resultados de riesgo.

Es importante considerar que para esta teoría la aparición de conductas de riesgo en adolescentes a partir de: la escasez de dinero y comodidad, la desigualdad y la discriminación que promueve parte de la sociedad, elementos que se pueden considerar a una parte de la población adolescentes como "riesgo", según el autor.

\section{Modelo sobre la comunicación familiar}

Definición: Olson (2000) conceptualiza que la comunicación familiar es una estrategia lo cual permite que se desarrolle habilidades para una comunicación asertiva o positivas, es calificada como un medio o canal que permite la facilitación para un buen funcionamiento familiar, permitiendo como resultado, niveles más adecuados de cohesión y adaptabilidad. De la misma manera la comunicación es un proceso de constante intercambio de información, ideas y emociones, entre el grupo familiar.

Modelos teóricos: Propuesta por Olson (2000), esta teoría se centra en las tres dimensiones centrales de sistemas maritales y familiares: cohesión, flexibilidad y comunicación.

La cohesión familiar se define como el vínculo emocional que los miembros de la familia tienen hacia el uno a otros, es decir el grado de la unión que existe entre ellos. Dentro de la cohesión los autores realizaron un diagnóstico para esto tomó, algunas consideraciones para medir las dimensiones que está conformado por; la vinculación emocional, limites, tiempo, espacio, relaciones intrapersonales, toma de decisiones e intereses y recreación. Los focos de la cohesión son como los sistemas que equilibran su separación frente a la unión. Presenta cuatro niveles, desde extremadamente bajo, moderado a extremadamente alto. Esta hipótesis de los niveles de cohesión central o equilibrada, hace referencia que la familia mantiene un buen funcionamiento, sin embargo, los niveles de desequilibrio son generalmente visto como problemático para las relaciones a largo plazo.

La flexibilidad familiar es la cantidad o la magnitud de cambio en su liderazgo, relaciones de roles y reglas. Los conceptos específicos incluyen; liderazgo (control y disciplina) negociación y estilos. El foco de la flexibilidad está en cómo los sistemas equilibran la estabilidad frente al cambio. La comunicación, es tomada como un punto muy importante dentro de la teoría, ya que es un elemento, lográndose la modificación en función a la dinámica de los matrimonios y familias. Es decir, modificando los estilos y estrategias de comunicación, se puede modificar el tipo de cohesión y flexibilidad que presentan. En las teorías mencionadas, se logra la jerarquía de la comunicación en el núcleo de la familia. Pues esto dependerá en la forma de cómo se logra comunicar los miembros de la familia, esto a su vez, determina su funcionamiento y la posibilidad de resolver o no los diferentes problemas que vayan presentando en el núcleo del sistema; por lo tanto, esto es el foco de su funcionalidad (Polaino, Lorente y Martínez, 2003).

Esta teoría sostiene que, mientas exista el funcionamiento esto ayudara a que se mantenga en balance, es decir la estructura de la familia podrá desarrollar la mayor posibilidad en cumplir las metas, objetivos y proyecciones, así se permitirá lograr que la familia cumpla las funciones (Ferrer. et al. 2013).

\section{METODOLOGÍA}

El diseño de la investigación es no experimental ya que no se manipularon las variables de estudio. Es de corte transversal, porque se recolectó la información en un determinado momento; y de tipo correlacional, debido a que se busca encontrar la relación entre la conducta sexual de riesgo y comunicación familiar

\section{Participantes}

La población fue de 500 alumnos, el tipo de muestreo fue probalísitico obteniendo así una muestra de 234 estudiantes, se encontró un $59.8 \%$ en el sexo masculino y un $40.2 \%$ en el sexo femenino, cuyas edades oscilan entre los 
13 y 19 años de ambos sexos, los alumnos que participaron fueron de 3 ro $(9,4 \%) 4$ to $(29,5 \%)$ y 5to $(61,1 \%)$ año del nivel secundario de una I. E. estatal de la ciudad de lquitos, a su vez dentro de las preguntas sociodemográfico los alumnos manifestaron que el $59,4 \%$ viven con sus padres, el $21,4 \%$ solo uno de ellos, el $17,1 \%$ con familias y un $2,1 \%$ viven con amigos.

\section{Instrumentos}

Los datos se obtuvieron mediante la aplicación de dos cuestionarios: el primero mide los niveles de conducta sexual de riesgo en adolescentes y el segundo evalúa la comunicación en el sistema familiar.

\section{Cuestionario de conducta sexual de riesgo}

La medición de los niveles de conducta sexual de riesgo en adolescentes, se elaboró un cuestionario por Apaza (2018) en Lima. Este cuestionario de conducta sexual de riesgo, puede administrarse de manera individual o colectiva, consta de 18 ítems con opciones de respuesta dicotómicas. Dicho cuestionario contiene 4 ítems orientadas en la iniciación sexual temprana en los estudiantes adolescentes, y 5 ítems encaminadas en la cantidad de parejas sexuales, como también 3 ítems están relaciones con el consumo de sustancias psicoactivas durante la conducta sexual de riesgo, a su vez esta 4 ítems relacionadas con el método de protección y por ultimo 2 ítems en conocimiento de las enfermedades de trasmisión sexual (ETS).

\section{Escala de comunicación Padres-Adolescentes (PAC).}

EstaescalafueelaboradaoriginalmenteporBarnes y Olson (1985) para evaluar la comunicación en el sistema familiar. Posteriormente adaptada por Bueno, Tomas y Araujo (1998) para adolescentes escolarizados de Lima. La escala de comunicación padres-adolescentes, puede administrarse de manera individual o colectiva, con un tiempo aproximando de 15 minutos. Presenta dos dimensiones; apertura en la comunicación y problemas en la comunicación. Esta escala presenta dos formatos: formato para el adolescente, con respecto a la madre y formato para el adolescente, con respecto al padre. Cada uno de los formatos está compuesto por 20 ítems, divididos en cada subescala compuesta de 10 ítems cada uno tasado en la escala de Likert de cinco puntos va desde uno (Nunca, Pocas veces, Algunas veces, Muchas veces, Siempre). Por otro lado, para la validación en el Perú, participaron 255 estudiantes (162 mujeres) de dos universidades de Lima. Cuyo resultado se obtuvieron altos valores de alfa de Cronbach (entre .80 y .90). Con ello se confirma la confiabilidad y la validez de este instrumento en la muestra investigada.

\section{Procesamiento de datos}

Una vez obtenido los datos, este se ingresó a una base de datos utilizando el SSPS. Por consiguiente, para establecer la asociación entre las variables entre las conductas sexuales de riesgo y la comunicación familiar se utilizó por el estadístico conveniencia Chi-cuadrado. También para el procesamiento de datos se realizaron las tablas de frecuencias obteniendo así un análisis descriptivo.

\section{RESULTADOS}

Se aprecia en la tabla 1 que el $60.3 \%$ de los adolescentes evaluados tuvo su primera relación sexual entre los 13 a 15 años de edad. Además, se observa que el $21.4 \%$ tuvo su primera relación sexual entre las edades de 16 a 18 años y el $18.4 \%$ tuvo su primera relación sexual entre los 10 a 12 años.

Tabla 1

Edad de inicio sexual de los adolescentes

\begin{tabular}{lll} 
Edad & N & $\%$ \\
\hline 10 a 12 años & 43 & $18.4 \%$ \\
13 a 15 años & 141 & $60.3 \%$ \\
16 a 18 años & 50 & $21.4 \%$ \\
\hline
\end{tabular}

En la tabla 2 se observa que solo el $30.8 \%$ de los adolescentes ha tenido una pareja sexual en toda su vida, mientras que el $43.6 \%$ ha tenido entre 2 a 4 parejas sexuales. También se aprecia que en los últimos seis meses si bien la mayoría solo ha tenido una pareja sexual $(70.5 \%)$, el $24.4 \%$ ha tenido entre 2 a 4 parejas sexuales. 
Tabla 2

Cantidad de parejas sexuales

\begin{tabular}{lllll} 
& \multicolumn{2}{l}{ Parejas sexuales en toda su vida } & \multicolumn{2}{l}{ Parejas sexuales en los últimos 6 meses } \\
\hline & $n$ & $\%$ & $n$ & $\%$ \\
1 pareja sexual & 72 & $30.8 \%$ & 165 & $70.5 \%$ \\
2 a 4 parejas sexuales & 102 & $43.6 \%$ & 57 & $24.4 \%$ \\
Más de 6 parejas sexuales & 60 & $25.6 \%$ & 12 & $5.1 \%$ \\
\hline
\end{tabular}

La tabla 3 muestra que menos de la mitad de los adolescentes utilizaron preservativo (43.2\%) como método de protección. Además, se aprecia que el $69.2 \%$ ha mantenido relaciones sexuales en los últimos seis meses y la mayoría no utilizo preservativos en su última relación sexual (54.3\%). También se observa que el $15.8 \%$ bebió alcohol y el $6 \%$ consumió alguna droga en la última vez que tuvieron relaciones sexuales. Se observa además que el $40.6 \%$ tuvo relaciones sexuales que conoció en ese día, el $34.2 \%$ tuvo relaciones sexuales con amigos en alguna fiesta y el $14.4 \%$ ha tenido relaciones sexuales en grupo. Finalmente se aprecia que el $21.4 \%$ de los adolescentes señala que ha tenido alguna enfermedad de transmisión sexual en los últimos seis meses.

Tabla 3

Conductas sexuales de riesgo

\begin{tabular}{|c|c|c|c|c|}
\hline & \multicolumn{2}{|c|}{ No } & \multicolumn{2}{|c|}{ Si } \\
\hline & $\mathbf{n}$ & $\%$ & $\mathbf{n}$ & $\%$ \\
\hline $\begin{array}{l}\text { ¿En esa primera relación sexual, usted o su pareja usó } \\
\text { condón? }\end{array}$ & 131 & $56 \%$ & 101 & $43.2 \%$ \\
\hline ¿Has tenido relaciones sexuales en los últimos 6 meses? & 72 & $30.8 \%$ & 162 & $69.2 \%$ \\
\hline $\begin{array}{l}\text { La última vez que tuvieron relaciones sexuales con pene- } \\
\text { tración, ¿tú o tu pareja usaron condón? }\end{array}$ & 127 & $54.3 \%$ & 107 & $45.7 \%$ \\
\hline $\begin{array}{l}\text { ¿Tomaste alcohol la última vez que tuviste relaciones se- } \\
\text { xuales? }\end{array}$ & 197 & $84.2 \%$ & 37 & $15.8 \%$ \\
\hline $\begin{array}{l}\text { ¿Consumiste alguna droga la última vez que tuviste rela- } \\
\text { ciones sexuales coitales? }\end{array}$ & 220 & $94 \%$ & 14 & $6 \%$ \\
\hline $\begin{array}{l}\text { ¿Alguna vez has tenido relaciones sexuales con una } \\
\text { persona que conociste ese día (en una fiesta, discoteca, } \\
\text { chat, etc.)? }\end{array}$ & 139 & $59.4 \%$ & 95 & $40.6 \%$ \\
\hline $\begin{array}{l}\text { ¿Estuviste bajo los efectos del alcohol cuando tuviste } \\
\text { relaciones sexuales con alguien que recién conociste? }\end{array}$ & 199 & $85 \%$ & 35 & $15 \%$ \\
\hline $\begin{array}{l}\text { ¿Has tenido relaciones sexuales con amigos(as)? (en } \\
\text { alguna fiesta, reunión, etc.) }\end{array}$ & 154 & $65.8 \%$ & 80 & $34.2 \%$ \\
\hline ¿Has tenido relaciones sexuales en grupo? & 201 & $85.9 \%$ & 33 & $14.1 \%$ \\
\hline $\begin{array}{l}\text { ¿Durante los últimos } 12 \text { meses ha tenido alguna Infección } \\
\text { de Trasmisión Sexual? }\end{array}$ & 184 & $78.6 \%$ & 50 & $21.4 \%$ \\
\hline $\begin{array}{l}\text { ¿Alguna vez ha tenido relaciones sexuales con una perso- } \\
\text { na, a la que tuvo que pagar por ello? }\end{array}$ & 197 & $84.2 \%$ & 37 & $15.8 \%$ \\
\hline $\begin{array}{l}\text { ¿Estuviste bajo los efectos del alcohol o alguna droga } \\
\text { cuando tuviste relaciones sexuales con una persona a la } \\
\text { que pagaste por ello? }\end{array}$ & 225 & $96.2 \%$ & 9 & $3.8 \%$ \\
\hline
\end{tabular}

Se aprecia en la tabla 4 que solo el $24.4 \%$ de los adolescentes evaluados tuvo relaciones sexuales bajo los efectos de alguna droga, como licor (17.9\%), cocaína (1.7\%), marihuana (1.3\%), PBC $(0.9 \%)$, Heroína $(0.9 \%)$ y otras drogas $(0.9 \%)$. 
Tabla 4

Relaciones sexuales estando bajo el efecto de alguna de las siguientes sustancias

\begin{tabular}{|c|c|c|c|c|}
\hline & \multicolumn{2}{|l|}{ No } & \multicolumn{2}{|l|}{ Si } \\
\hline & $n$ & $\%$ & n & $\%$ \\
\hline Nunca he tenido relaciones sexuales bajo los efectos de alguna droga & 57 & $24.4 \%$ & 177 & $75.6 \%$ \\
\hline Licor, Alcohol & 192 & $82.1 \%$ & 42 & $17.9 \%$ \\
\hline Cocaína & 230 & $98.3 \%$ & 4 & $1.7 \%$ \\
\hline Marihuana & 231 & $98.7 \%$ & 3 & $1.3 \%$ \\
\hline PBC (Pasta Básica de Cocaína) & 232 & $99.1 \%$ & 2 & $0.9 \%$ \\
\hline Tranquilizantes/ Pastillas para los nervios & 232 & $99.1 \%$ & 2 & $0.9 \%$ \\
\hline Éxtasis, LSD & 232 & $99.1 \%$ & 2 & $0.9 \%$ \\
\hline Heroína & 232 & $99.1 \%$ & 2 & $0.9 \%$ \\
\hline Terokal & 232 & $99.1 \%$ & 2 & $0.9 \%$ \\
\hline Otros & 232 & $99.1 \%$ & 2 & $0.9 \%$ \\
\hline
\end{tabular}

Se aprecia en la tabla 4 que no existe asociación significativa entre el nivel de comunicación con el padre y las conductas sexuales de riesgo. En cambio, se aprecia que el nivel de comunicación con la madre se asocia significativamente con la edad de inicio sexual $(X 2=2.303 ; p<.05)$, con la cantidad de parejas sexuales $(X 2=8.758 ; p<.01)$ y uso de preservativo $(X 2=3.803 ; p<.05)$ como medida de protección.

Tabla 5

Asociación entre comunicación con los padres y las conductas sexuales de riesgo

\begin{tabular}{lllllll} 
& \multicolumn{3}{l}{ Comunicación con el padre } & \multicolumn{3}{l}{ Comunicación con la madre } \\
\hline & $\mathrm{X}^{2}$ & $\mathrm{G}$ & $\mathrm{P}$ & $\mathrm{X}$ & $\mathrm{gl}$ & $\mathrm{p}$ \\
Edad de inicio sexual & 2.303 & 4 & .680 & 9.744 & 4 & .045 \\
Cantidad de parejas sexuales & 8.758 & 4 & .067 & 13.918 & 4 & .008 \\
Consumo de drogas & 1.310 & 2 & .520 & 4.916 & 2 & .086 \\
Uso de preservativo & 3.803 & 4 & .433 & 11.106 & 4 & .025 \\
\hline
\end{tabular}

Se aprecia en la figura 1 que los adolescentes que iniciaron sexualmente entre 16 a 18 años a su vez tienen una buena comunicación con la madre. También se aprecia que un nivel de comunicación regular y deficiente se asocia con una edad de inicio sexual más temprano.

Figura 1

Asociación entre edad de inicio sexual y comunicación con la madre

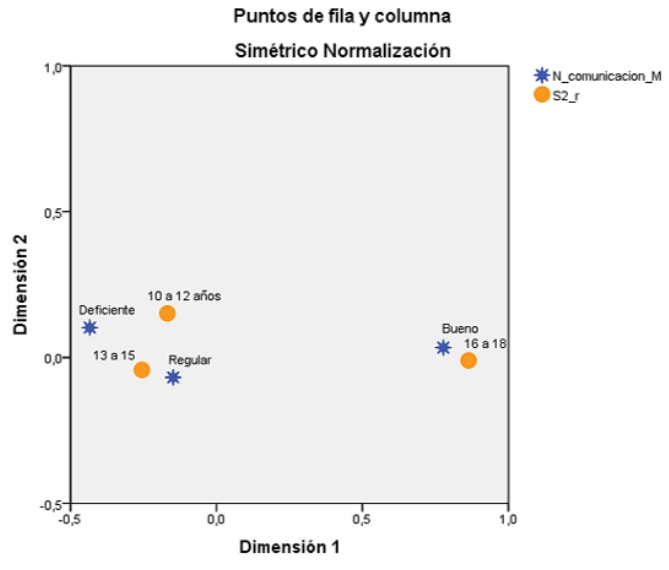


Se aprecia en la figura 2 que una deficiente comunicación con la madre se asocia a un mayor número de parejas sexuales por parte de los adolescentes (2 a 5 parejas sexuales) y una buena comunicación se asocia a tener una sola pareja sexual.

Figura 2

Asociación entre número de parejas sexuales y comunicación con la madre

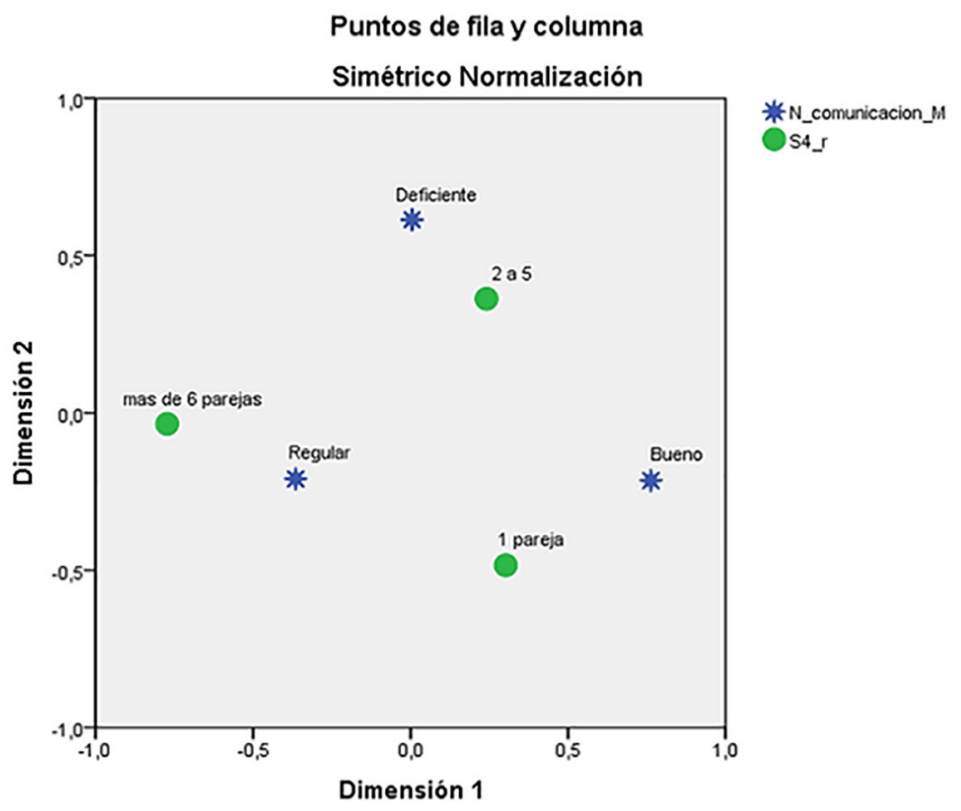

Se aprecia en la figura 3 que el uso ocasional del preservativo por parte del adolescente se asocia a una comunicación regular con la madre y los adolescentes que mantienen una comunicación deficiente con la madre a su vez nunca utilizan preservativo.

\section{Figura 3}

Asociación entre uso de preservativo y comunicación con la madre

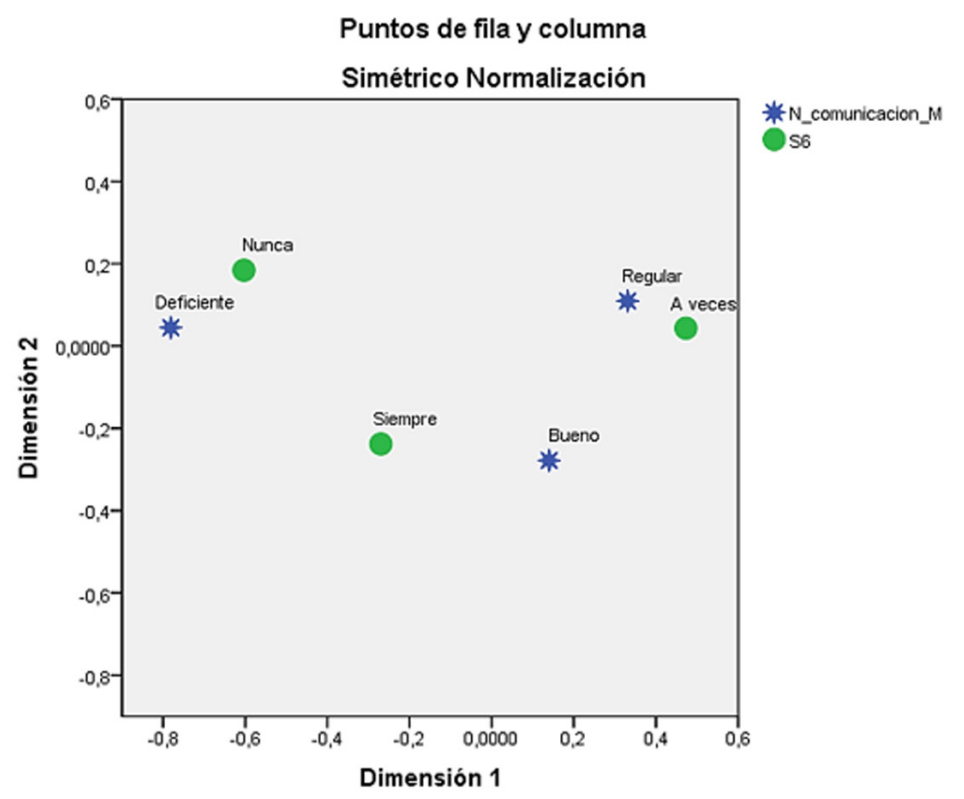




\section{DISCUSIÓN}

En los últimos años la conducta sexual de riesgo se ha incrementado de manera notable en la amazonia peruana, debido a que los adolescentes dan inicio a su actividad sexual a temprana edad, en consecuencia de una comunicación escasa con los padres, asimismo, Montoya, Castaño y Moreno (2016) hacen referencia que los niños y jóvenes de hoy están creciendo con la ausencia física del padre, pues la realidad evidencia que el padre actual manifiesta otro tipo de acompañamiento y crianza, esto se debe a que el padre está priorizando la economía del hogar, sus aspiraciones personales en el trabajo, entre otras cosas, de este modo el vínculo afectivo padre e hijo es deficiente. En la investigación, se pudo observar que, en nuestra muestra, la composición familiar es más que estructurada $(59,4 \%)$ y monoparental $(40,6 \%)$.

En relación a ello, el objetivo del estudio fue determinar si existe relación significativa entre la conducta sexual de riesgo y la comunicación de los padres con los hijos. Para lo cual se evaluó por separado la comunicación de los padres, una evaluación separada, que permite una mejor comprensión de la comunicación. Ya que según Urías (2015) menciona que la comunicación juega un rol muy importante del núcleo familiar, siendo así, la comunicación categorizada como abierta, ofensiva o evitativa, existiendo así una diferenciación en la toma de estilos de crianza entre el padre y la madre.

En este sentido, los resultados encontrados muestran asociación entre la comunicación con la madre y conducta sexual de riesgo, es decir, que cuanto mayor sea la comunicación con la figura materna menor será el inicio de conductas sexuales en los adolescentes. El resultado coincide con lo expuesto por Cisneros y Palacios (2018) en su investigación hallaron que independientemente el tipo de comunicación que el adolescente tenga con el padre no influye de manera significativa en las conductas sexuales de riesgo, Sin embargo, en cuanto a la comunicación de los adolescentes con su madre se evidencia que tienen mayor probabilidad de tener una conducta sexual de riesgo: iniciar precozmente sus relaciones coitales, más de una pareja sexual, cambio frecuente de parejas, relaciones fugaces y prácticas de sexo "oro genital/genito - anal sin protección". Por su parte Uribe, Castellanos y Cabán (2016) manifiestan que hay una tendencia de los adolescentes a conversar con mayor facilidad acerca de temas referente a la sexualidad, con la figura materna. Del mismo modo, Almanza y Ccencho (2016) en su investigación tuvo como resultado que los adolescentes tienen mejor apertura con la madre que con el padre, así mismo, se muestran en los datos que los adolescentes del sexo masculino y femenino muestran mayor confianza en la comunicación con la madre que con el padre. Concordado también con Granda (2015) que indica que los adolescentes tienen una comunicación abierta con la madre a diferencia del padre. Por lo tanto, la comunicación con la madre es significativa para el adolescente, debido a que pospone el inicio de las conductas sexuales de riesgo. Por otro lado, no se evidencia asociación significativa entre comunicación con el padre y conducta sexual de riesgo, que incluye, inicio temprano de las relaciones sexuales, no usar métodos de protección, múltiples parejas y el uso de sustancias psicoactivas. Estos resultados coinciden con Schonfled, McReey y Eisenberg (2013) que deducen en su estudio que existe diversas causas que pueden influir entre la comunicación entre padres e hijos, uno de ellos es el género, denotando la figura materna con mayor seguridad, confianza y responsabilidad a la hora de hablar de sexualidad, respondiendo así las dudas que tienen los adolescentes a diferencia del padre que se muestra más rígido. Teniendo, así como resultado que la comunicación con el padre no influye de manera significativa en las conductas sexuales de riesgo.

Respecto a la comunicación con la madre y la edad de inicio sexual se encontró que existe una relación significativa $(X 2=2.303 ; p<.05)$, donde los adolescentes que tienen una mejor comunicación con su madre su inicio sexual es a mayor edad (16 a 18 años) mientras que los adolescentes que tienen dificultades en la comunicación con su madre su inicio sexual es a menor edad (10 a 12 años). Mientras exista una buena comunicación con la madre, la edad de inicio sexual será a mayor edad. Correlacionado al estudio Uribe at al (2013) manifiesta que existe diversos estudios en las cuales afirma que la conducta sexual aumenta cada vez más, se reportan el gran incremento de la iniciación sexual temprana, permitiendo que los adolescentes den un inicio precoz en la formación de hogares. Acotando a esto Salazar et al (2007) hace una referencia que, en las zonas rurales del Perú, los adolescentes dan inicio a temprana edad su actividad sexual ya que, se presenta una escasa comunicación respecto a la educación sexual y al cuidado de su cuerpo 
del adolescente, demostrando a esto un clima familiar poco expresiva en afecto y comunicación. En este sentido, Gamarra y lannacone (2009), concluye que diversos estudios mencionan que los adolescentes que viven en familias estructuras o completas tienen mayor probabilidad en retrasar su actividad sexual, en cambio, sí solo se presencia la figura materna o paterna el inicio será aún más temprano.

Del mismo modo, se encontró una relación altamente significativa entre la cantidad de parejas sexuales y la comunicación con la madre $(X 2=8.758 ; p<.01)$, lo cual hace referencia que, solo el $30.8 \%$ de los adolescentes ha tenido una pareja sexual en toda su vida, mientras que el $43.6 \%$ ha tenido entre 2 a 4 parejas sexuales. Así mismo, en los últimos seis meses si bien la mayoría solo ha tenido una pareja sexual (70.5\%), el $24.4 \%$ ha tenido entre 2 a 4 parejas sexuales. Es decir, entre más alta sea la comunicación con la madre menor cantidad de parejas sexuales en los adolescentes. Fortaleciendo a esto Parra y Oliva (2002) manifiestan que, en estos casos, los adolescentes perciben a las madres más abiertas en comunicarse, comprensivas e interesantes al hablar de sexualidad, llevándose a iniciar una comunicación intercambiada. Por otro lado, Rafael (2016) en su investigación realizada en Perú concluye que, los adolescentes procedentes de familias con disfunción familiar tuvieron en mayor frecuencia de dos a más parejas sexuales en comparación a adolescentes de familias funcionales, es decir aquellos adolescentes que tenían de dos a más parejas estaban predispuestos a contraer una enfermedad de trasmisión sexual. Por otra parte, Arias et al., (2011) menciona que los hombres son los que tienen mayor frecuencia a presentar mayor número de parajes, las cuales en su mayoría de veces son ocasionales, permitiendo que se presente con mayor índice en vulnerabilidad.

Respecto al resultado no existe una asociación significativa entre el consumo de sustancias psicoactivas y la comunicación con la madre, (X2=4.916; $p<.09$ ), es decir, que la comunicación que tiene el adolescente con la figura materna no influye en el consumo de sustancias psicoactivas. De los jóvenes encuestados que llevan a cabo prácticas orientadas al consumo riesgoso de alguna droga, siendo el licor el de más alto consumo, seguido por la cocaína, la marihuana y en bajas proporciones el PBC entre otras drogas. En otra investigación realizada por Apaza y Vega (2015) podemos encontrar que si bien no todos los jóvenes encuestados en dicho estudio, realizan prácticas orientadas a conductas peligrosas específicamente el consumo de sustancias psicoactivas, por lo tanto, se concluyó que el consumo de las bebidas alcohólicas y el uso de drogas en adolescentes no presentaron una asociación significativa en el estudio realizado.

Esto difiere con la investigación realizada por Bordalejo, Schmidt, Molina y Sartuqui (2016) mostrando en su estudio que no todos los adolescentes tienen prácticas riesgosas con respecto al consumo de alcohol, sin embargo, el $20 \%$ de su muestra, indican que la comunicación con la madre resulta significativa solo para las mujeres (hijas adolescentes). Se ha encontrado que la restricción en la comunicación se asocia a prácticas sociales vinculadas a un consumo riesgoso de alcohol. A su vez, una comunicación positiva se asocia a una menor orientación a tales prácticas.

Finalmente se encontró una asociación entre las conductas sexuales de riesgo y comunicación con la madre siendo el más común el uso del preservativo $(X 2=3.803 ; p<.05)$, frente a ello, se puede deducir que entre más buena sea la comunicación con la madre, más probabilidades que los adolescentes utilice preservativos. Esto es coherente con los resultados de la presente investigación, donde menos de la mitad de los adolescentes utilizaron preservativo (43.2\%)como método de protección. En el estudio de Lavielle, et. al. (2014) evaluaron las conductas sexuales de riesgo del adolescente (la iniciación de la vida sexual, múltiples parejas y la no utilización del condón) y su relación con algunas características y el tipo de dinámica familiar (expresión del afecto y la comunicación) y la satisfacción con la familia. Siendo la comunicación y la calidez entre de padres e hijos, un rol de suma importancia, ya que influye como protectores en las conductas sexuales de riesgo del adolescente. Por otro lado, Gonzales (2016) manifiesta que realizaron investigaciones relacionadas con la comunicación y la sexualidad, lo cual concluyeron que, las conversaciones que se daban entre padres e hijos se centran en abordajes basados en el miedo referente a los embarazos no deseados y las enfermedades de trasmisión sexual, pero dentro de ello poco se relacionan en establecer comunicación sobre experiencias sexuales, englobando a esto los adolescentes se relacionan con más eficacia con la madre ya que mantienen una conversación de valores y reglas que trasmite una orientación moral para la protección y uso de 
preservativos en los adolescentes. Por su parte Forcada et. al (2013) alude que mientras mejor es la comunicación entre padres e hijos, mayor uso de preservativos entre los adolescentes. Así mismo, mencionan que la comunicación sexual con los padres, no es suficiente, sino que la calidad general de la comunicación podría ser la variable relevante en las conductas sexuales de riesgo.

\section{Declaración de financiamiento y de conflictos de interés:}

El estudio fue financiado por los autores, quienes declaran no tener conflictos de interés.

\section{Correspondencia}

Keshia Gamboa Pumazoncco

Correo electrónico:

keshiagamboa@upeu.edu.pe

Keila Ysuiza Rodríguez

Correo electrónico:

keila.ysuiza@gmail.com

\section{REFERENCIAS BIBLIOGRÁFICAS}

Almanza, R. \& Ccencho, E. (2016) Comunicación familiar y aborto en adolescentes que se atienden en el hospital regional de Zacarias Correa Valdivia de Huancavelica Enero a Octubre del 2016. (Tesis para optar el título profesional de obstetra, universidad Nacional de Huancavelica) recuperado de: http://repositorio.unh.edu. pe/bitstream/handle/UNH/1143/TP-UNH. OBST.00067. pdf?sequence=1\&isAllowed

Apaza, L. y Vega, E. (2018) Factores personales y sociales relacionados con el inicio de la actividad sexual en estudiantes de una institución educativa (Lima, Perú). Disponible en: http:// www.federacion-matronas.org/wp-content/ uploads/2018/07/Original-breve_Factorespersonales.pdf

Arias, L. M., Vázquez, M., Dueñas, E., García, L., \& Tejada, E. (2011). Comportamiento sexual y erotismo en estudiantes universitarios, Cali, Colombia. Colombia Medica, 42(3), 309-318. Recuperado de http://www.redalyc.org/comocitar. oa?id=28322503007.

Bordalejo, M., Schmidt, V., Molina, M. y Sartuqui, A. (2016). Comunicación con los padres y su relación con el consumo de alcohol en la adolescencia. VIII Congreso Internacional de Investigación y Práctica Profesional en Psicología XXIII Jornadas de Investigación XII Encuentro de
Investigadores en Psicología del MERCOSUR. Facultad de Psicología - Universidad de Buenos Aires, Buenos Aires. Disponible en: https://www. aacademica.org/000-044/261.pdf

Fernández, A., Negrón, M., González, L., Díaz, I., Betancourt-Díaz, E., Cintrón-Bou, F., Varas-Díaz, N., \& Villarruel, A. (2017). Actitudes hacia la comunicaciónentrepadres, madresyadolescentes en Puerto Rico. Revista puertorriqueña psicología; 28 (1): 80-95. Recuperado de: http://europepmc.org/backend/ptpmcrender. fcgi?accid=PMC5520656\&blobtype=pdf

Ferrer, P., Miscán, Ana., Pino, M., \& Pérez, V. (2013). Funcionamiento familiar según el Modelo Circumplejo de Olson en familias con un niño que presenta retardo mental. Revista. Enfermeria Herediana, 6(2), 51-58. Recuperado de http://faenf.cayetano.edu.pe/images/pdf/ Revistas/2013/febrero/funcionamientofamiliarse gunelmodelocircumplejodeolson.pdf

Forcada, P., Pacheco, A., Pahua, E., Pérez, P., Todd, N., \& Pulido, M. (2013). Conducta sexual de riesgo en estudiantes universitarios: Factores de riesgo y protección. Revista Intercontinental de Psicología y Educación, 15(1), 23-46. Recuperado de: http:// www.redalyc.org/pdf/802/80225697003.pdf

Granda, M. (2015). Comunicación entre padres e hijos y su influencia en las conductas del riesgo en la unidad educativa fiscal misional Domingo Seli del canton catacocha periodo 2014- 2015. (Tesis para la obtención de licenciado de ciencias educación, Universidad Nacional del Loja, Ecuador)

Gómez, M. (2013). Conocimientos y actitudes frente a las conductas sexuales de riesgo en adolescentes (Tesis para Optar el grado de Licenciada en Enfermería, Universidad Nacional Jorge Basadre Grohmann, Tacna). Recuperado de: http://repositorio.unjbg.edu. pe/bitstream/handle/UNJBG/220/TG0071. pdf? sequence $=1$ \&isAllowed

Gonzales, V. (2016). Comunicación familiar y toma de decisiones en sexualidad entre ascendientes y adolescentes. Revista latinoamericana ciencias sociales niñez juventud, 15 (1): 419-430. Recuperado de: http://revistaumanizales.cinde. org.co/rlcsnj/index.php/Revista-Latinoamericana/ article/view/2972/805

Hernández. R., Fernández. C., \& Baptista. M., (2010). Metodología de la Investigación 5ta Edición. México: McGraw.Hill.

Jessor, R. (1991). Risk behavior in adolescence: A psychosocial framework for understanding and action. Journal of Adolescent Health, 12, 597605. Recuperado de: https://www.colorado. edu/ibs/jessor/pubs/1991_Jessor_JAH_ RiskBehaviorinAdolescence.pdf 
Jessor, R. (1993). Desarrollo adolescente exitoso entre jóvenes en entornos de alto riesgo. American Psychologist, 48, 117-126. Recuperado de: https://www.colorado.edu/ibs/ jessor/pubs/1993_Jessor_AmerPsychologist_ SuccessfulAdolescentDevelopment.pdf

Lavielle, P., Jiménez, F., Vázquez, A., Aguirre, M., Castillo, M., \& Vega, S. (2014). Impacto de la familia en las conductas sexuales de riesgo de los adolescentes. Revista Médica del Instituto Mexicano del Seguro Social, 52(1), pp. 38-43 Instituto Mexicano del Seguro Social Distrito Federal, México. Recuperado de: http://www. redalyc.org/pdf/4577/457745480010.pdf

Montoya, Z., Castaño, N. y Moreno, N. (2016). Enfrentando la ausencia de los padres: recursos psicosociales y construcción de bienestar. Revista Colombiana de Ciencias Sociales, 7(1), 181-200. Recuperado de: https://dialnet.unirioja. es/servlet/articulo?codigo $=5454158$

Olson, D. H. Barnes, H. L., \& (1985). Parent-adolescent communication and the circumplex model. Child development, 438-447. Recuperado: https:// www.jstor.org/stable/1129732?seq=1\#page_ scan_tab_contents

Olson, D. H. (2000). Circumplex Model of Marital and Family Systems. Journal of Family Therapy, 22(2), 144-167. Recuperado: https://doi. org/10.1111/1467-6427.00144

Parra, A. y Oliva, A. (2002). Comunicación y conflicto familiar durante la adolescencia. Revista anales de psicología .18, Pag. 215-231. Recuperado por https://revistas.um.es/analesps/article/ download/28421/27521/0

Polaino-Lorente, A. \& Martínez, P. (2003). Evaluación Psicológica y Psicopatológica de la familia. Madrid. Ediciones: Rialp. Recuperado por: https:// books.google.com.pe/books?id=10FLqwoq1X QC\&lpg=PA23\&dq= Polaino

Rafael, M. (2016). Relación entre funcionalidad familiar y comportamiento sexual de riesgo en adolescentes del Instituto Materno Perinatal en el periodo febrero de 2016. (Tesis para optar título profesional de Médico Cirujano, Universidad Mayor De San Marcos, Lima). Recuperado por http://cybertesis.unmsm.edu. pe/bitstream/handle/cybertesis/4696/Rafael_ $\mathrm{cm}$.pdf? sequence $=1$ \&isAllowed $=\mathrm{y}$

Rosales, J., \& Irigoyen, A. (2013). Embarazo en adolescentes: problema de salud pública y prioridad para el médico familiar. Editorial atención familiar, 20 (4), 101-102.

Salazar, A., et al. (2007). Conocimiento de sexualidad, inicio de relaciones sexuales y comunicación familiar, en adolescentes de instituciones educativas nacionales del distrito de el Agustino, Lima-Perú. Revista horizonte medico. 7 (2), 79-85. Recuperado por http://www.horizontemedicina. usmp.edu.pe/index.php/horizontemed/article/ view/214/229. presentar\%20ultimo

Urías, K. (2015). Relación de la comunicación familiar y la victimización escolar de adolescentes. Revista de investigación y práctica en psicología del desarrollo, 109-117. Recuperado de: https://revistas.uaa.mx/index.php/ippd/article/ view/637/614

Uribe, J., et al. (2013). Predictores del uso del condcon en las relaciones sexuales de adolescentes, análisis diferencial por sexo. Revista internacional de Psicologia y educación. Mexico Pag. 7592. Recuperado por https://www.redalyc.org/ $\mathrm{html} / 802 / 80228344005 /$

Uribe, A., Castellanos, J. y Cabán, M. (2016)Conductas sexuales de riesgo y comunicación sobre sexualidad entre padres e hijos universitarios. DOI: 10.17533/udea.rpsua.v8n2a03 Disponible en: file://C:/Users/user/Downloads/DialnetCon ductasSexualesDeRiesgoYComunicacionSobre Sexuali-6059401\%20(6).pdf

Valle, M., Benavides, R., Álvarez, A., \& Peña, J. (2011). Conducta sexual de riesgo para $\mathrm{VIH} /$ SIDA en jóvenes universitarios. Revista de Enfermería Inst. Mex. Seguro Soc; 19 (3): 133136. Recuperado de: http://www.medigraphic. com/pdfs/enfermeriaimss/eim-2011/eim113d.pdf

Recibido: 05/08/2020

Aceptado: 15/10/2020 